\title{
Sympathetic skin responses in hemiplegic patients with and without complex regional pain syndrome
}

\author{
Barin Selçuk, Murat Ersoz, Murat Inanir, Aydan Kurtaran, Müfit Akyuz \\ $1^{\text {st }}$ PM and R Clinic, Ankara Physical Medicine and Rehabilitation Education and Research Hospital of Ministry of Health, \\ Ankara, Turkey
}

Background and Aims: To investigate whether there were changes in the sympathetic skin responses (SSR) in the limbs with complex regional pain syndrome (CRPS) type I in hemiplegic patients. Setting: A physical medicine and rehabilitation center in Turkey. Materials and Methods: Sympathetic skin responses were evaluated in 69 stroke patients ( 41 with CRPS and 28 without CRPS) and 20 healthy volunteers. SSR were recorded on the paretic and healthy hands after stimulation of the ipsilateral median nerve. Patients' ages ranged from 33 to 77 years, with a mean of $60.0 \pm 12.9$ years. Results: The SSR were obtained in all patients with CRPS, whereas SSR was absent in 9 of 28 patients with hemiplegia who did not have CRPS after stimulation of the plegic side and the difference was statistically significant $(P=0.023)$. SSR amplitudes were increased at the hemiplegic limbs in patients affected by CRPS compared to individuals unaffected; this group difference was statistically significant $(P=0.014)$. The mean amplitude of the SSR in the advanced stage of CRPS was greater than lower stage and the difference was statistically significant $(P=0.035)$. Conclusion: Our results suggest that SSR can be obtained in stroke patients with CRPS even in the early stages of CRPS. SSR acquirability and amplitude increase as the stage of the disease advances. As an electrophysiologic technique, SSR may be used in the evaluation of the sympathetic function in hemiplegic patients and also in the diagnosis of CRPS and in monitoring of its treatment.

Key words: Autonomic dysfunction, complex regional pain syndrome, hemiplegia, stroke, sympathetic skin responses

\section{Introduction}

Complex regional pain syndrome (CRPS) type I, a persistent painful shoulder disability with homolateral pain and swelling of the hand, is not a rare complication in patients with hemiplegia and often presents a serious barrier to successful stroke rehabilitation because of patient discouragement. ${ }^{[1]}$ It is characterized by a chronic painful condition of the upper limb associated with neurovascular disturbance and dystrophic changes of the skin and bone. It is believed to involve longstanding autonomic nervous system disturbance caused by brain damage. ${ }^{[2]}$ However, there is no one unifying theory of the pathophysiology of CRPS that adequately explains all of its features.

The disease process ordinarily progresses through different stages. Stage I is characterized by significant pain and increased blood flow, with resultant pitting edema, redness and warmth. Reduced range of motion of wrist, hand and shoulder often begins to develop at this stage. The skin can be hyperesthetic and tender. In stage II, the edema is often less prominent; however, pain and further impairment in range of motion are prominent features. Blood flow is decreased and the temperature of the extremity falls. Stage III is characterized by less pain, progression to irreversible changes of atrophic soft tissue, muscle atrophy, severe loss of motion and excessive osteoporosis. ${ }^{[3]}$

The diagnosis is ordinarily made on a clinical basis, primarily with the use of history and observation and the physical signs. Technetium diphosphonate bone scan has been used as a sensitive and specific test to objectively ascertain the presence of CRPS. ${ }^{[4]}$ This technique may become the gold standard for diagnosing CRPS but has several disadvantages, which include radiation exposure to relative invasiveness and high cost.

Sympathetic skin response (SSR) studies are frequently used in clinical electrophysiology laboratories to evaluate autonomic nervous system functions. ${ }^{[5]}$ This technique records changes in skin conductance after activation of sweat glands in skin areas rich in ecrine glands under the neural control of sympathetic sudomotor fibers. ${ }^{[6]}$ Potentials generated by the SSR can be recorded in response to various stimuli; these include electrical peripheral nerve stimulation, acoustic stimuli or magnetic stimulation of nerves of the brain. ${ }^{[7,8]}$

If CRPS is a result of sympathetic overactivity, then we would 
expect the SSR to be increased in the affected limbs. So this test may serve as an objective measure of the presence of CRPS and may be more practical than bone scans as an initial diagnostic procedure. $^{[2]}$

The aim of the study was to investigate whether there were changes in SSR in the limbs with CRPS. We also looked for any relationship between different stages of CRPS and SSR. The final goal was to decide whether this test was used in the evaluation of the sympathetic function in hemiplegic patients and also in the diagnosis and monitoring of the treatment of CRPS.

\section{Materials and Methods}

The ethics committee of our hospital approved the study protocol and informed consent was obtained from the subjects. This prospective study was performed on 69 hemiplegic or hemiparetic stroke patients (43 males and 26 females with an average age of $60.0 \pm 12.9$ years) and 20 healthy volunteers (13 males and 7 females with an average age of $57.9 \pm 15.6$ years) at our hospital between 2000 and 2005. Patients with manifestations of other central or peripheral nervous system lesions and patients and healthy subjects with any other diseases known to affect the autonomic nervous system (e.g., diabetes, alcoholism) or those receiving medications that affect autonomic nervous system function were excluded. Disease duration was 161 days in the patients with CRPS and 139 days in the patients without CRPS. Computed tomography revealed ischemic infarcts in 56 patients (81\%), hemorrhagic infarcts in 13 patients (19\%). Thirty-two patients had left hemiplegia, whereas 37 patients had right hemiplegia.

Patients diagnosed with CRPS in upper limb using Kozin's clinical criteria were recruited. ${ }^{[9]}$ Forty-one of them had CRPS, whereas 28 of them did not. None of the patients with CRPS had prior treatment of the disease and all had sustained cerebrovascular accident that was presumed causal to the development of CRPS. All patients were informed about the aim and method of the study and informed consent was obtained from the patients or their legal guardian.

The experiment was performed under standard condition in an illuminated and silent room with the temperature kept at 24 to $25^{\circ} \mathrm{C}$. Limbs were covered during the test with blankets in order to maintain palmar temperature between 34 and $36^{\circ} \mathrm{C}$. During the testing, all patients were kept awake and relaxed in the supine position.

Recordings were carried out using a 4-channel Dantec keypoint electromyographic device. SSR were recorded from paretic hands using disc electrodes, $6 \mathrm{~mm}$ in diameter, positioned on the volar and dorsal surfaces. To evoke the SSR, the median nerve was stimulated at the wrist. SSR were recorded on the paretic and healthy hands after stimulation of the ipsilateral median nerve in hemiplegic patients. To avoid any habituation, stimulations were made with randomized intervals of various intensities. The duration of the stimulus was between 0.1 and $0.3 \mathrm{~ms}$ and the stimulus intensity ranged from 20 to $50 \mathrm{~mA}$. Sensitivity was set at $500 \mathrm{~V} /$ div and time at $500 \mathrm{~ms} /$ div. Low frequency filter was set at $0.2 \mathrm{~Hz}$ and high frequency filter at $500 \mathrm{~Hz}$. At least five responses from both hands were recorded after stimulation and were superimposed. If a response to 10 consecutive stimuli was not evident, SSR were presumed absent. Onset latency, amplitude and the number of the phases were recorded for all responses in each limb. Latencies were measured from onset of the first deflection of the signal from baseline and amplitudes were measured from peak to peak. Statistical analyses were performed with student's t-test, $\chi^{2}$ test and Kruskal Wallis test by using statistical package for social sciences (SPSS).

\section{Results}

Forty-one patients had CRPS, whereas 28 did not. All patients had normal median and ulnar nerve conduction studies. SSR were obtained in all patients with CRPS, whereas SSR was absent in $9(32 \%)$ of the 28 without CRPS after stimulation of the plegic side and the difference was statistically significant $(P=0.023)$. Also, SSR was absent in 7 of the $69(10 \%)$ hemiplegic patients after stimulation of the healthy side and there was no significant difference between the SSR amplitudes $(P=0.078)$ and latencies $(P=0.094)$ recorded at plegic and healthy sides of the patients [Table 1].

The comparison of SSR obtained from the hemiplegic patients with CRPS and without CRPS showed that the amplitude of SSR in patients with CRPS was significantly higher than in patients without CRPS $(P=0.014)$, but there was no significant difference between the SSR latencies recorded in patients with CRPS and without CRPS $(P=0.089)$. Table 2 shows the results of the SSR recording in patients with and without CRPS [Figure 1].

The amplitude of the SSR in hemiplegic patients with CRPS increased as the stage of the disease advanced. In other words, the amplitudes of SSR in CRPS stage III was higher than in CRPS stage I and II; and in CRPS stage II, it was higher than stage I and the difference was statistically significant $(P=0.035)$, but there was no significant difference between the SSR latencies recorded in patients with CRPS stages. Table 3 shows the results of SSR recordings of patients with CRPS in each stage.

Table 1: Comparison of sympathetic skin response amplitude and latency values in plegic and healthy sides of the hemiplegic patients

$\begin{array}{ccc}\begin{array}{c}\text { Hemiplegic } \\ \text { side }(\mathbf{n}=69)\end{array} & \begin{array}{c}\text { Healthy } \\ \text { side }(\mathbf{n}=69)\end{array} & \boldsymbol{P} \\ 1024 \pm 574 & 1179 \pm 690 & 0.078 \\ 1.67 \pm 0.51 & 1.58 \pm 0.44 & 0.094\end{array}$

Table 2: Comparison of sympathetic skin response amplitude and latency values in hemiplegic patients with CRPS and without CRPS

$\begin{array}{ccc}\begin{array}{c}\text { Hemiplegic } \\ \text { patients with }\end{array} & \begin{array}{c}\text { Hemiplegic } \\ \text { patients without }\end{array} & \boldsymbol{P} \\ \text { CRPS }(\mathbf{n}=\mathbf{4 1}) & \text { CRPS }(\mathbf{n}=\mathbf{2 8}) & \\ 1046 \pm 550 & 695 \pm 640 & 0.014 \\ 1.70 \pm 0.38 & 1.82 \pm 0.41 & 0.089\end{array}$


Table 3: Comparison of sympathetic skin response amplitude and latency values in hemiplegic patients with CRPS in each stage

\begin{tabular}{|c|c|c|c|c|c|}
\hline \multirow[b]{3}{*}{ Amplitude $(\mu \mathrm{V})$} & \multirow{2}{*}{$\begin{array}{l}\text { Hemiplegic patients without CRPS (n:28) } \\
\text { No CRPS ( } n: 28)\end{array}$} & \multicolumn{3}{|c|}{ Hemiplegic patients with CRPS (n:41) } & \multirow[t]{2}{*}{$\boldsymbol{P}$} \\
\hline & & Stage I (n:19) & Stage II (n:12) & Stage III (n:10) & \\
\hline & $695 \pm 640$ & $897 \pm 301$ & $1001 \pm 324$ & $1296 \pm 350$ & 0.035 \\
\hline Latency (sec) & $1.82 \pm 0.41$ & $1.78 \pm 0.30$ & $1.55 \pm 0.23$ & $1.73 \pm 0.28$ & 0.077 \\
\hline
\end{tabular}

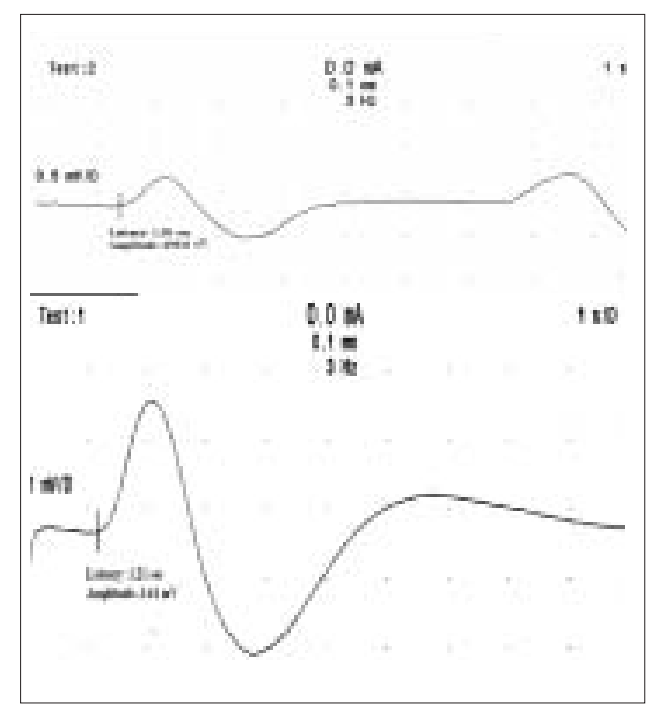

Figure 1: Sympathetic skin responses recorded in patients with CRPS stage III (lower) and without CRPS (upper)

There was no significant difference between the SSR amplitudes $(P=0.125)$ and latencies $(P=0.145)$ recorded in patients with hemorrhagic and ischemic stroke.

The comparison of SSR obtained from the patients and healthy subjects showed that the amplitude of SSR in plegic side of patients was significantly lower $(P=0.015)$ and the latencies of SSR were significantly shorter than healthy subjects $(P=0.021)$ [Table 4].

\section{Discussion}

We found that the amplitude of SSR in patients with CRPS was significantly higher than in patients without CRPS, but there was no significant difference between the SSR latencies recorded in patients with CRPS and without CRPS. SSR were obtained in all patients with CRPS, whereas SSR was absent in $32 \%$ of the patients without CRPS after stimulation of the plegie side. Similar to our findings, many studies have reported amplitude abnormalities of SSR in hemiparetic extremities during different stages of stroke. ${ }^{[2,5,10-11]}$ Overactivity of the sympathetic system, as seen in CRPS, would be expected to alter skin resistance through its effect on sudomotor fibers, thereby effecting the amplitude of

Table 4: Comparison of sympathetic skin response amplitude and latency values in plegic side of patients and healthy volunteers

\begin{tabular}{lccc}
\hline & \multicolumn{2}{c}{$\begin{array}{c}\text { Plegic side of } \\
\text { patients }(\mathbf{n}=69)\end{array}$} & $\begin{array}{c}\text { Healthy } \\
\text { volunteers }(\mathbf{n}=\mathbf{2 0})\end{array}$ \\
Amplitude $(\mu \mathrm{V})$ & $1024 \pm 574$ & $2006 \pm 972$ & 0.015 \\
Latency $(\mathrm{sec})$ & $1.67 \pm 0.51$ & $1.31 \pm 0.31$ & 0.021 \\
\hline
\end{tabular}

the SSR. Due to the complex and multisynaptic nature of SSR, it can be affected by CRPS independent of its pathogenesis, However, very few studies have compared the responses in patients with and without CRPS. In the study by Clinchot et al, in four patients with CRPS comparing the SSR amplitude and latency in the same subject between the hemiplegic side and healthy side, the amplitudes of the hemiplegic sides significantly increased and the latencies significantly shortened. In their study, they used the uninvolved extremities as the control. The authors speculated that the higher amplitude and the shorter latency response from the hemiplegic side after stimulating the extremity with CRPS is because the stimulus given is not appropriately transferred centrally as a result of the cerebrovascular accident that the patient had experienced and that leads to lower contralateral response. ${ }^{[2]}$

We also revealed that the amplitude of the SSR in hemiplegic patients with CRPS increased as the stage of the disease advanced. In other words, the amplitude of SSR in CRPS stage III was highest and the amplitude of the SSR in stage I was lowest and there was no significant difference between the SSR latencies in patients with different CRPS stages. Increased sympathetic activity, which is claimed for the etiology of CRPS, may be present from the onset of cerebrovascular accident and as a result, SSR responses may be obtained from patients with CRPS; more advanced investigation is warranted on this topic. In early stage of the stroke, sympathetic sudomotor dysfunction may be related to the absent or diminished excitatory or modification effect of cortical structures on the sympathetic nervous system, which increased in CRPS, especially in the advanced stages secondary to increased sympathetic overactivity. In this study, although the number of the patients in stage II and III was small (12, 10 respectively), the fact that the SSR amplitudes increased with increasing CRPS stage lead us to speculate that SSR may be used in the early diagnosis of CRPS, in determining the efficacy of treatment modalities and in the follow-up of treatment.

Alterations of sympathetic outflow is a well-known consequence of hemispheric or brainstem damage. ${ }^{[12]}$ The central localization of SSR is not well known. It is thought that the mesencephalic reticular formation and posterior hypothalamus play an important role in the formation of this reflex. The cerebral cortex plays an important role in the modification of the response. This arrangement is claimed to be made through the corticoreticular tracts. ${ }^{[11]}$ It is thought that the cerebral cortex demonstrates its inhibitory and excitatory effects on the sudomotor sympathetic activity on the contralateral side of the body. But the exact mechanism and the localization of the effective cortical areas are not known. It is thought that the increase in sweating and conductivity of the skin in patients with a cortical lesion is due to 
the disappearance of the cortical inhibitory effect on the sudomotor sympathetic system. ${ }^{[13]}$

Today an SSR evaluation as 'the response is present or absent' is accepted as sufficient because of large variations in latencies and amplitudes. ${ }^{[14-15]}$ Our study design controlled some factors, such as temperature, habituation. Recently, opinions emphasizing the necessity to take into consideration the amplitudes and the latency differences between contralateral sides and between the upper and lower extremities have become more important. ${ }^{[16]}$ However, abnormalities of SSR latencies and amplitudes or asynchrony of the response has been suggested as a criterion for abnormalities. ${ }^{[17-18]}$ Although there are difficulties in standardization of SSR studies, especially in amplitudes of the responses, it seems possible to use this method in the early diagnosis of CRPS, in determining an effective treatment and in the evaluation of treatment efficacy.

It is possible that after cerebrovascular accident, especially in the presence of CRPS, sympathetic hyperactivity appears and as a result, SSR could be obtained from these patients even in each stage of CRPS. SSR amplitude increased as the stage of the disease advanced. As an electrophysiologic technique, SSR may be used in the evaluation of the sympathetic function in hemiplegic patients. It is particularly important in post-stroke patients because the differentiation of CRPS from the other peripheral causes for regional pain (such as periarthritis, arterial occlusion, etc.) is not easy. And this technique may also be useful in the diagnosis of CRPS and in evaluation of the efficacy of the treatment.

\section{References}

Eto F, Yoshikawa M, Ueda S, Hirai S. Posthemiplegic shoulder-hand svndrome, with special reference to relation cerebral localization. J Am Geriat Soc 1980;28:13-7.
2. Clinchot DM, Lorch F. Sympathetic skin response in patients with reflex sympathetic dystrophy. Am J Phys Med Rehabil 1996;75:252-6.

3. Strakowski JA, Wiand JW, Johnson EW. Upper limb musculoskeletal pain syndromes. Braddom RL (editor): Physical Medicine and Rehabilitation. WB Saunders: Philadelphia; 2000. p. 792-817.

4. Mackinnon SE, Holder LE. Use of three-phase radionuclide bone scanning in diagnosis of reflex sympathetic dystrophy. J Hand Surg 1984;9:556-63.

5. Zimmerman KP, Monga TN, Darouiche RO, Lawrence SA. Post-stroke autonomic nervous system function: Palmar sympathetic skin responses thirty or more days after cerebrovascular accident. Arch Phys Med Rehabil 1995;76:250-6.

6. Rossini PM, Opsomer RJ, Boccasena P. Sudomotor skin responses following nerve and brain stimulation. Electroencephalogr Clin Neurophysiol 1993;89:442-6.

7. Balci N, Balci NK, Beyazova M. Sympathetic skin response and R-R interval variation in diabetic patients. J Rheumatol Med Rehabil 1998;9:166-71.

8. Akyuz G, Sozuer D'T, Turan B, Canbolat N, Yýlmaz I, Us O, et al. Normadative data of sympathetic skin response and $\mathrm{R}-\mathrm{R}$ interval variation in Turkish children. Brain Dev 1999;21:9-102.

9. Kozin F. Painful shoulder and reflex sympathetic dystrophy syndrome, in Mc Carty DJ (editor): Arthritis and Allied Conditions: A Textbook of Rheumatology, $10^{\text {th }} \mathrm{ed}$. Lea and Febiger: Philadelphia; 1985. p. 1322-55.

10. Muslumanoglu L, Aki S, Turkdogan D, Us O, Akyuz G. Involvement of sympathetic reflex activity in patients with acute and chronic stroke: A comparison with functional motor capacity. Arch Phys Med Rehabil 2004;85:470-3.

11. Korpelainen JT, Tolonen U, Sotaniemi KA, Myllyla VV. Suppressed sympathetic skin response in brain infarction. Stroke 1994;24:1389-92.

12. Linden D, Berlit P. Sympathetic skin responses (SSRs) in monofocal brain lesion: Topographical aspect of central sympathetic pathways. Acta Neurol Scand 1995:91:372-6.

13. Cechetto DF, Saper CB. Role of the cerebral cortex in autonomic function. In Loewy AD, Spyer KM editors. Central regulation of autonomic function. Oxford University Press: New York; 1990. p. 208-23.

14. Roth EJ, Harevey RL. Rehabilitation of stroke syndromes. In: Randall L Braddom (editor): Physical Medicine and Rehabilitation. WB Saunders Company: Philadelphia; 1996. p. 1053-87.

15. Walker SM, Cousins M.J. Complex regional pain syndrome: Including reflex sympathetic dystrophy and causalgia. A Review. Anaesth Intens Care 1997;25:11325 .

16. Shahani BT, Halperin JJ, Boulu P, Cohen J. Sympathetic skin response: A method of assessing unmyelinated axon dysfunction in peripheral neuropathies. J Neurol Neurosurg Psychiatr 1984;47:536-42.

17. Toyokura M, Murakami K. Reproducibility of sympathetic skin response. Muscle Nerve 1996;19:1481-3.

18. Barron SA, Rogovski Z, Hemli J. Autonomic consequences of cerebral hemisphere infarction. Stroke 1994;25:113-6.

Accepted on 18-05-2006 